\title{
Short communication: Ultrasonographic assessment of lung consolidation postweaning and survival to the first lactation in dairy heifers
}

\author{
E. A. Adams*1 and S. Buczinski† \\ *Dairy Technical Services Team, Merck Animal Health, 35500 W 91st Street, De Soto, KS 66018 \\ †Département des sciences cliniques, Faculté de Médecine Vétérinaire, Université de Montréal, CP5000, Saint-Hyacinthe, J2S 7C6, \\ Québec, Canada
}

\begin{abstract}
The aim of this prospective cohort study was to assess the association of systematic thoracic ultrasonography findings postweaning on calves' survivability to the first lactation. Three-month-old Jersey heifers $(\mathrm{n}=250)$ returning from a custom heifer grower were scanned by thoracic ultrasonography and lungs assessed using a scoring system with a scale from 1 to 4 . A score of 1 was attributed to calves with no abnormality. A score of 2 was assigned if multiple comet tails or B-lines (coalescence of multiple comet tails) were observed. A score of 3 was assigned to calves with $\geq 1$ location of lung consolidation $\geq 1 \mathrm{~cm}$ but $<6 \mathrm{~cm}$. Calves with extensive consolidation ( $\geq 6 \mathrm{~cm}$ in one or more locations) or evidence of abscessation or pleural effusion $(>1 \mathrm{~cm})$ were assigned a score of 4 . Calves with a score of 4 had greater risk of dying or being culled $[26 \%(95 \%$ credibility interval: $13-47 \%)]$ than calves with a score of $1[1 \%(0-6 \%)], 2[3 \%(1-9 \%)]$, or $3[5 \%(1-17 \%)]$. We found no association between age of first calving in the remaining calves and lung score. Thus, lung lesion severity assessed by thoracic ultrasound is associated with a long-term production outcome.
\end{abstract}

Key words: dairy calf, lung consolidation, ultrasonography, pneumonia

\section{Short Communication}

Bovine respiratory disease complex (BRD) continues to be a challenge for dairy producers and custom calf raisers (USDA-APHIS, 2011). During a pneumonic event, damage to the lung from the pathogen itself or due to the calf's own immune response can causes consolidation, fibrosis, effusion, and abscessation (Panciera and Confer, 2010). Evidence indicates that these lesions

Received August 14, 2015.

Accepted October 19, 2015.

${ }^{1}$ Corresponding author: elizabeth.adams@merck.com have negative effects on performance in cattle as seen by the decreased ADG of beef cattle with lungs lesions found at slaughter compared with cattle without lung lesions (Wittum et al., 1996). A study of South African feedlot cattle found that the overall effect of BRD, defined by lung lesions measured at slaughter, was a 24-g reduction in ADG and a 5.1-d increase in days on feed (Thompson et al., 2006). Calves with a history of being treated for pneumonia during calfhood have an increased age at first calving and are likely to make less milk in the first lactation and be culled within the first lactation (Bach, 2011; Stanton et al., 2012). On many calf-raising operations, treatment records are not permanent and the pneumonia diagnosis is highly subjective (Van Donkersgoed et al., 1993; Sivula et al., 1996; McGuirk, 2008). Therefore, the true effect of pneumonia is still not well defined because most of the previous studies have relied on the farm's treatment recording of disease incidence, which is based on different subjective clinical impressions and imperfect case definitions. Among more specific tools for BRD detection, thoracic ultrasound allows the operator to image the lung surface. Ultrasound can serve as an objective tool for the diagnosis of BRD-induced lung damage compared with necropsy (Braun et al., 1997; Rabeling et al., 1998; Flöck, 2004). Thoracic ultrasound can also be used calf-side on the farm (Ollivett et al., 2011) and is easily and consistently performed, even for novice operators with proper training (Buczinski et al., 2013). The accuracy of thoracic ultrasound for BRD detection in calves has recently been determined with a sensitivity of $79.4 \%$ [95\% Bayesian credibility interval (BCI) of $66.4-90.9 \%]$ and a specificity of $93.9 \%(95 \%$ BCI: 88.0-97.6\%; Buczinski et al., 2015). The objective of this study was to determine if fixed-time lung ultrasound findings could be applied on farm to predict whether calves have increased odds of not entering the productive state (first lactation). Our hypothesis was that pathologic ultrasonographic lung anomalies are associated with a negative outcome of not entering the first lactation and eventually delayed age at first calving (AFC). 
Three-month-old Jersey heifers returning from a custom heifer grower were selected as candidates for this study. The calves were from 3 different source farm locations in California that were under the same ownership and management strategies. They left their home dairies on d 2 of life and were raised by a custom grower who raised calves for multiple other dairies in addition to the calves enrolled in this study. Treatment records and specifics of the feeding program while the calves were at the custom calf-growing operation were unavailable. Upon reaching 3 mo of age, the calves left the custom calf grower and returned to a single heifergrowing operation operated by the owner of the calves. At 6 mo of age, the calves were grouped and transported to Texas to continue their heifer development and breeding. Pregnant heifers returned to California as springers when they were 7 mo pregnant with their first calf. For the purposes of this study, record keeping began when the calves were 3 mo old and returned under the management of ownership until freshening.

The returning 3-mo-old calves were grouped (55-60 calves) in an open dirt corral with a roof, cement lane, and 60 appropriately sized locking head stanchions. The calves were vaccinated with a modified live viral with 5-way leptospira vaccine (Bovishield Gold 5L5, Zoetis Animal Health, New York, NY) upon returning to the dairy at 3 mo of age. A booster vaccine was administered 4 wk later. No metaphylaxis or group treatment with antibiotics was given to the animals when they returned at 3 mo of age. Upon returning from the custom calf raiser and for the duration of the study, the calves were evaluated for illness twice a day by a trained on-farm employee. On the day of enrollment, every other calf in a pen was unlocked and released from the head stanchion. The remaining calves were scanned with the ultrasound one time, a lung score assigned, and their performance followed to first lactation. A total of 250 calves were enrolled in this study over 2-mo period based on the availability of animals returning. Calves were delivered to the heifer growing operation every 2 wk in groups of 60 to 90 calves depending on the number of calves that met the minimum age requirement of $90 \mathrm{~d}$. Calves were enrolled and their lungs scanned the day after they returned from the custom grower.

The thoracic lung ultrasound technique was performed as previously described (Babkine and Blond, 2009) with the substitution of acoustic gel for isopropyl alcohol. Briefly, hair on both sides of the thorax was clipped from the tenth rib space cranially to the third rib space and isopropyl alcohol applied to the skin. A 8.5-MHz linear transducer ultrasound (Ibex Pro, E.I. Medical Imaging, Loveland, CO) was used in the intercostal spaces where the hair was clipped to image the lung. The maximal depth of examination was set at 8 $\mathrm{cm}$ throughout the study period. The probe was moved from the caudal to the cranial lung fields, moving in each intercostal space from dorsal to ventral. Confirmation of viewing the entire lung field was made by imaging the liver on the right and spleen on the left in the caudal regions to the heart and lung cranial to the heart on the right and left sides in the cranial regions. The same operator (EA) scanned all of the calves that were enrolled in this study.

A simple scoring system that aimed to be compatible with a rapid calf-side use was developed to assess calf lung lesions. A calf was scored on a scale of 1 to 4 based on findings within the entire lung field as follows. A score of 1 was attributed to calves with no abnormalities, meaning only a healthy pleural surface or isolated comet tail within an image field could be seen (Figure 1A). A score of 2 was given if comet tails on the pleural surface that were multiple and B-lines (coalescence of multiple comet tails; Lichtenstein et al., 2004) without significant lung consolidation were observed (Figure 1B). A score of 3 was assigned to calves with one or more locations of lung consolidation $\geq 1 \mathrm{~cm}$ (Figure 1C). A score of 4 was assigned to calves with extensive consolidation $(\geq 6 \mathrm{~cm}$ in one or more locations), abscessation within the lung parenchyma seen as an encapsulated fluid filled space (Figure 1D), or significant pleural effusion $(>1 \mathrm{~cm}$; Figure 1E).

The calf lung ultrasound score was recorded as an item in the calf's individual Dairy Comp (Valley Agricultural Software, Tulare, CA) record. Future vaccinations, treatments, inseminations, pregnancy diagnosis, freshening, and dead events were also recorded in the calf's individual record. When an animal was sold for voluntary reasons, this was also recorded.

The analyses were performed using commercial software (SAS software, version 9.3; SAS Institute Inc., Cary, NC). Descriptive statistics were performed concerning the distribution of lesions scores and the mortality and culling proportions. The association between ultrasonographic findings and the risk of being removed from the herd, voluntarily or involuntarily, was investigated using a nonparametric Kaplan-Meier survival analysis (LIFETEST procedure in SAS) log-rank test and post-hoc Tukey-Kramer adjustments for multiple comparisons between lung scores. Because almost all the mortality or culling events occurred before $330 \mathrm{~d}$ of life, we focused on this specific period for survival analysis.

The AFC was compiled for all calves. The relation between AFC and lung score was assessed using a nonparametric Wilcoxon rank-sum test (NPAR1WAY procedure in SAS). The level of $\alpha$ (type I) error was set at $<0.05$ and a tendency was reported $0.05 \leq \alpha<0.10$. 

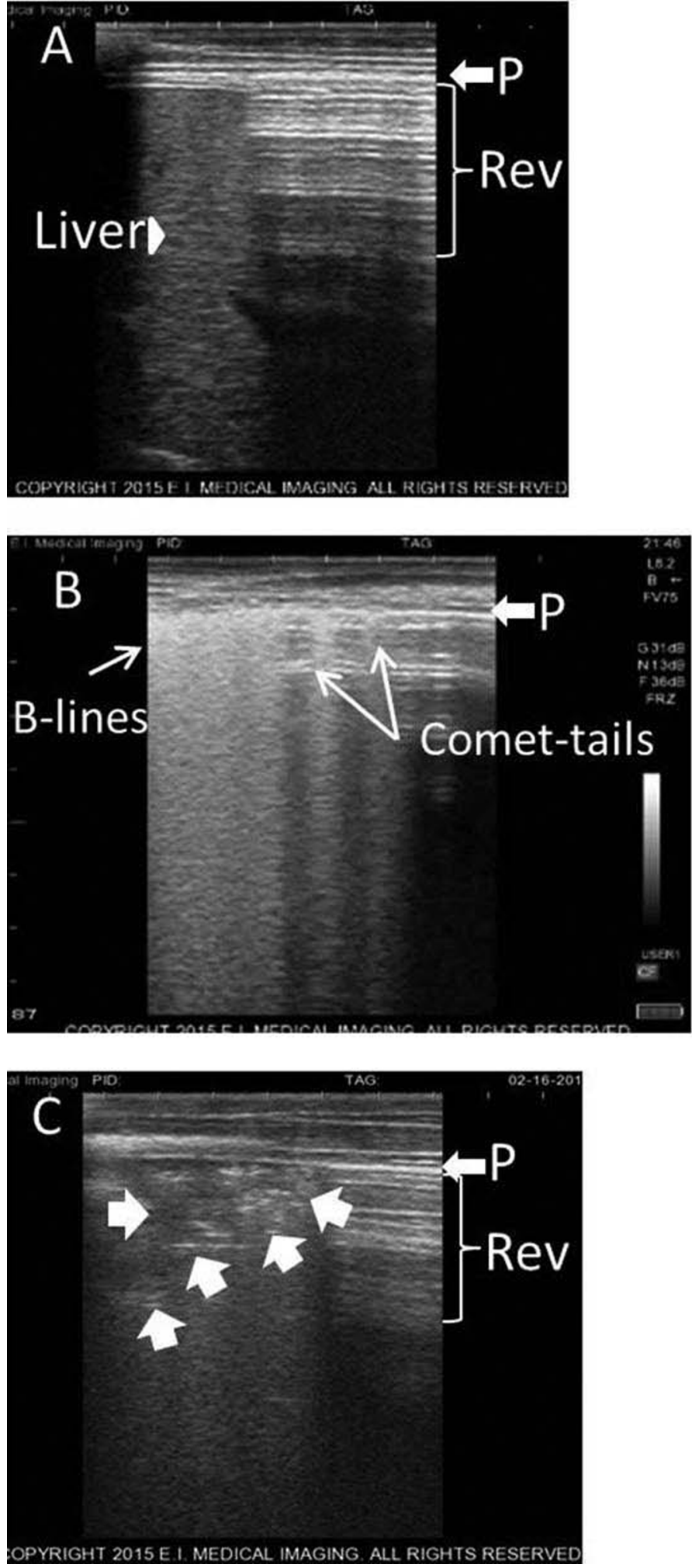

Figure 1. Gradation of thoracic ultrasonographic lesions in dairy calves. Scores: $1=$ no abnormalities, the reverberation artifact (Rev) allows observation of A-lines (reverberation lines; panel A); 2 = multiple comet tails (arrows in B) on the pleural surface or B-lines (coalescence of multiple comet tails without significant lung consolidation; panel B); $3=$ one or more location of lung consolidation $\geq 1 \mathrm{~cm}$ but $<6 \mathrm{~cm}$ (delineated with arrows in panel C); $4=$ at least one site with $\geq 6 \mathrm{~cm}$ of consolidation, abscessation within the lung parenchyma (panel D), or significant pleural effusion ( $>1 \mathrm{~cm}$; panel $\mathrm{E}$ ), where $\mathrm{P}=$ pleural line, and Plef $=$ pleural effusion.
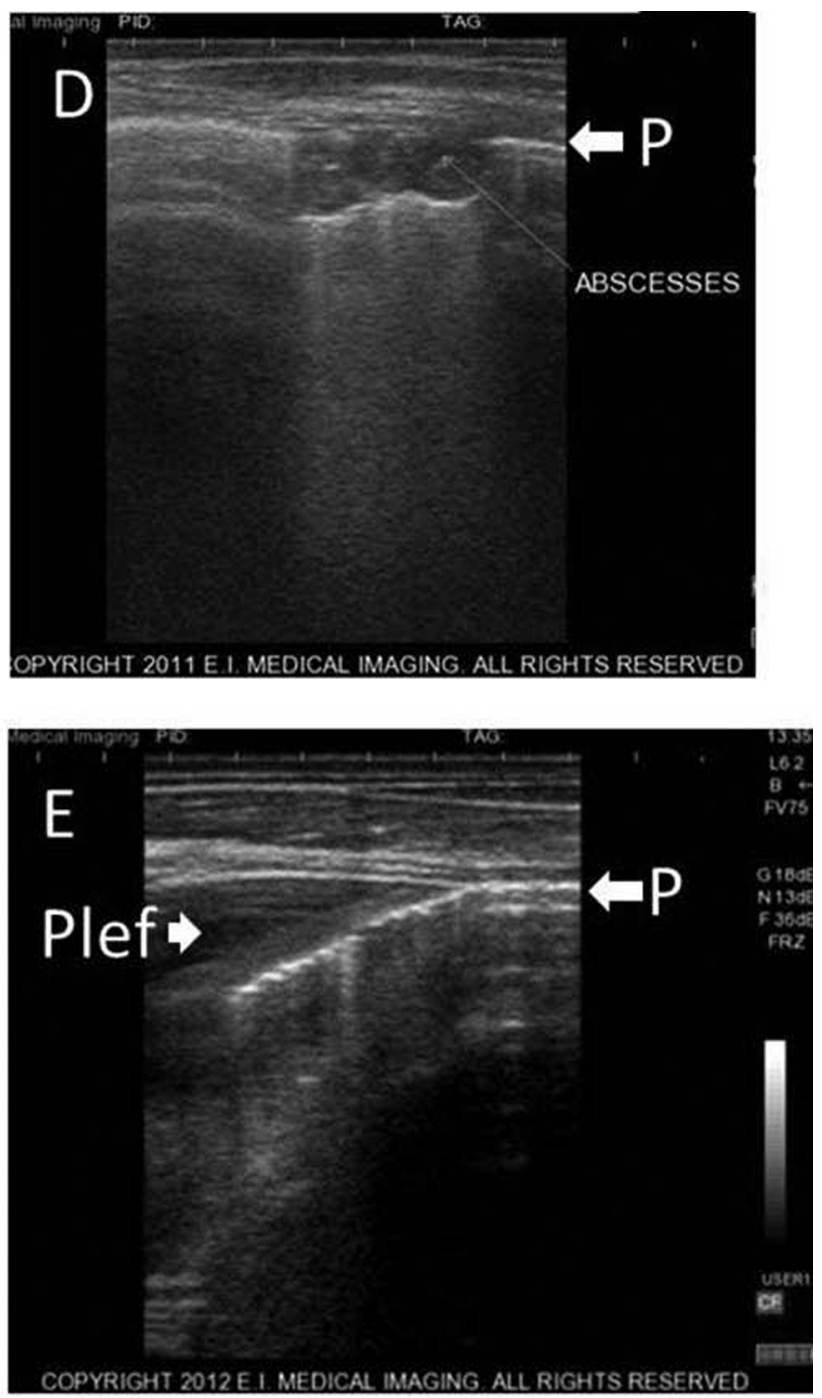
Table 1. Proportion of removal in Jersey dairy heifers depending on their ultrasonographic lung lesions postweaning

\begin{tabular}{|c|c|c|c|c|}
\hline Lung score ${ }^{1}$ & $\begin{array}{l}\text { Survived } \\
\quad \text { (no.) }\end{array}$ & $\begin{array}{l}\text { Removed } \\
\text { (no.) }\end{array}$ & $\begin{array}{c}\text { Probability of } \\
\text { removal }^{2}(95 \% \text { CI })\end{array}$ & $\begin{array}{l}\text { Total } \\
\text { (no.) }\end{array}$ \\
\hline 1 & 96 & 1 & $0.01(0-0.06)$ & 97 \\
\hline 2 & 74 & 2 & $0.03(0.01-0.09)$ & 76 \\
\hline 3 & 37 & 2 & $0.05(0.01-0.17)$ & 39 \\
\hline 4 & 20 & 7 & $0.26(0.13-0.47)$ & 27 \\
\hline Total & 227 & 12 & & 239 \\
\hline
\end{tabular}

${ }^{1}$ Score of $1=$ no abnormalities; $2=$ multiple comet tails on the pleural surface or B-lines (coalescence of multiple comet tails without significant lung consolidation); $3=$ one or more location of lung consolidation $\geq 1 \mathrm{~cm}$ but $<6 \mathrm{~cm} ; 4=$ at least one site with $\geq 6 \mathrm{~cm}$ of consolidation, abscessation within the lung parenchyma, or significant pleural effusion $(>1 \mathrm{~cm})$.

${ }^{2}$ Probability of removal $=$ proportion of the removed animals and all animals with the same lung score.

A total of 250 calves were examined during the study period (age at enrollment was $96 \pm 6 \mathrm{~d}$; mean $\pm \mathrm{SD}$ ), and final data were not available in 11 cases ( 5 calves with a lung score of 1,4 with a lung score of 2 , and 2 with a lung score of 3 ). These calves were therefore not included in the results. The descriptions of the remaining calves' lung scores and the proportion of removal following ultrasonographic examination are shown in Table 1.

The median time of death or culling due to poor performance was $45 \mathrm{~d}$ after examination (135 d of age; range 104-755 d). Calves with a lung score of 4 had a significant difference in survival compared with score 3 or score 1 or $2(P<0.01)$. We detected a tendency for calves with a score of 1 or 2 to have better survival than calves with a score of $3(P=0.06$; Figure 2$)$. We found no difference in AFC for calves with a lung score of 1 (median: $22.6 \mathrm{mo}$; range 21.2-26.9 mo), 2 (22.7 mo; 21.5-24.5 mo), 3 (22.1 mo; 20.1-24.5 mo), or 4 (22.6 mo; 21.4-25.8 mo; $P=0.5)$.

Bovine respiratory disease remains a challenge for young calves. The USDA National Animal Health Monitoring Service (NAHMS) data from 1991 to 2011 shows that the percentage of mortality from respiratory disease remained consistent at 21.3 to $24.5 \%$ in preweaned calves and increased slightly, from 38.4 to $46.5 \%$, in weaned calves (USDA-APHIS, 2011). Part of the challenge with managing pneumonia in young calves is that the case definition is subjective (Sivula et al., 1996). The objective value of rectal temperature has been shown to be an unreliable prognostic indicator in beef calves with suspicion of BRD (Theurer et al., 2014). Therefore, the true incidence of the disease and subsequent management decisions are not always clear. Thoracic ultrasound is a tool with which to make an objective diagnosis of BRD that could be applied as a screening exam to determine the risk of pneumonia contributing to the risk of involuntary culling in the first year of life.
This study showed that a single-timepoint thoracic ultrasound exam was associated with a decreased chance of survival in the first year of age but not with increased AFC. It is interesting to note that most of the heifers were removed rapidly after the ultrasonographic examination (median $45 \mathrm{~d}$ after the examination). This finding may be associated either with reactivation of pneumonia after being moved to their custom grower due to this stressful event or with poor performance due to the effect of lung lesion on growth, as shown in feedlot calves (Thompson et al., 2006). In contrast to the results in the current study, previous research has shown that the increased number of pneumonia cases in calves after $60 \mathrm{~d}$ of age is associated with an increased AFC (Stanton et al., 2012). A difference in the current study was that the extent of lung lesions, not recorded pneumonia events, was measured as a proxy for previous pneumonia disease. The ultrasound score in this study was assigned independently of the clinical signs of pneumonia or course of disease in the calves and there is evidence that lung consolidation diagnosed by ultrasound can become smaller over time, indicating that lungs can heal (Ollivett et al., 2013). Calves with the most severe lung lesions diagnosed at the singletimepoint thoracic ultrasound exam were significantly less likely to survive to first lactation and therefore a subset of the population was excluded from the AFC analysis. The lack of a difference in AFC in the remaining heifers could also be due to the fact that animals in this study were Jersey heifers, which have a reputation for high fertility (Garcia-Peniche et al., 2005), or because $4.4 \%(11 / 250)$ of cases did not have follow-up available. Milk production data for the heifers enrolled in this study were not available for analysis but milk production would be a valuable parameter to measure in future studies.

Calves returning from a custom grower to the home dairy operation often do not return with treatment records indicating health events that occurred early in 
life. Even if a calf remains under the control of a dairy owner from birth until first lactation, health events are often not permanently recorded and the subjective nature of diagnosing pneumonia can lead to unknowns when trying to understand the respiratory health of a herd. The length of time that lesions are present in the lungs will depend on when the pneumonia was diagnosed and treated, how well the calf responded to the treatment, and the type of pathogen involved. In a challenge study where calves were challenged intratracheally with Mannheimia haemolytica and lung consolidation was tracked over time, lesions first appeared $2 \mathrm{~h}$ after challenge, peaked in area of involvement 48 $\mathrm{h}$ after challenge, and could be visualized for up to 6 mo (Ollivett et al., 2013). Thoracic ultrasound gives a snapshot picture of the pathologic changes in the lungs as a consequence of BRD and, because the lesions last for a relatively long time, it is a tool that can be utilized by veterinarians to diagnose pneumonia.
In this study, the timepoint chosen for examination was upon return from a custom grower because the calves had unknown treatment histories. The data show that there was a population of calves with severe lung lesions and that these calves had a higher hazard of not surviving to freshening. A large range was found for the age at which animals were removed, but if nonproductive animals could be identified accurately earlier and removed from the herd sooner, there would be benefits of saved days on feed, saved money on future pneumonia treatments, and therefore improved herd profitability and improved animal welfare. Alternatively, specific management tools could be applied to improve the healing process if the calf has a good pedigree or understand why a calf may not live up to her genomic potential.

Another important step in applying thoracic ultrasound to BRD research would be to know how to differentiate active versus inactive lung lesions in the

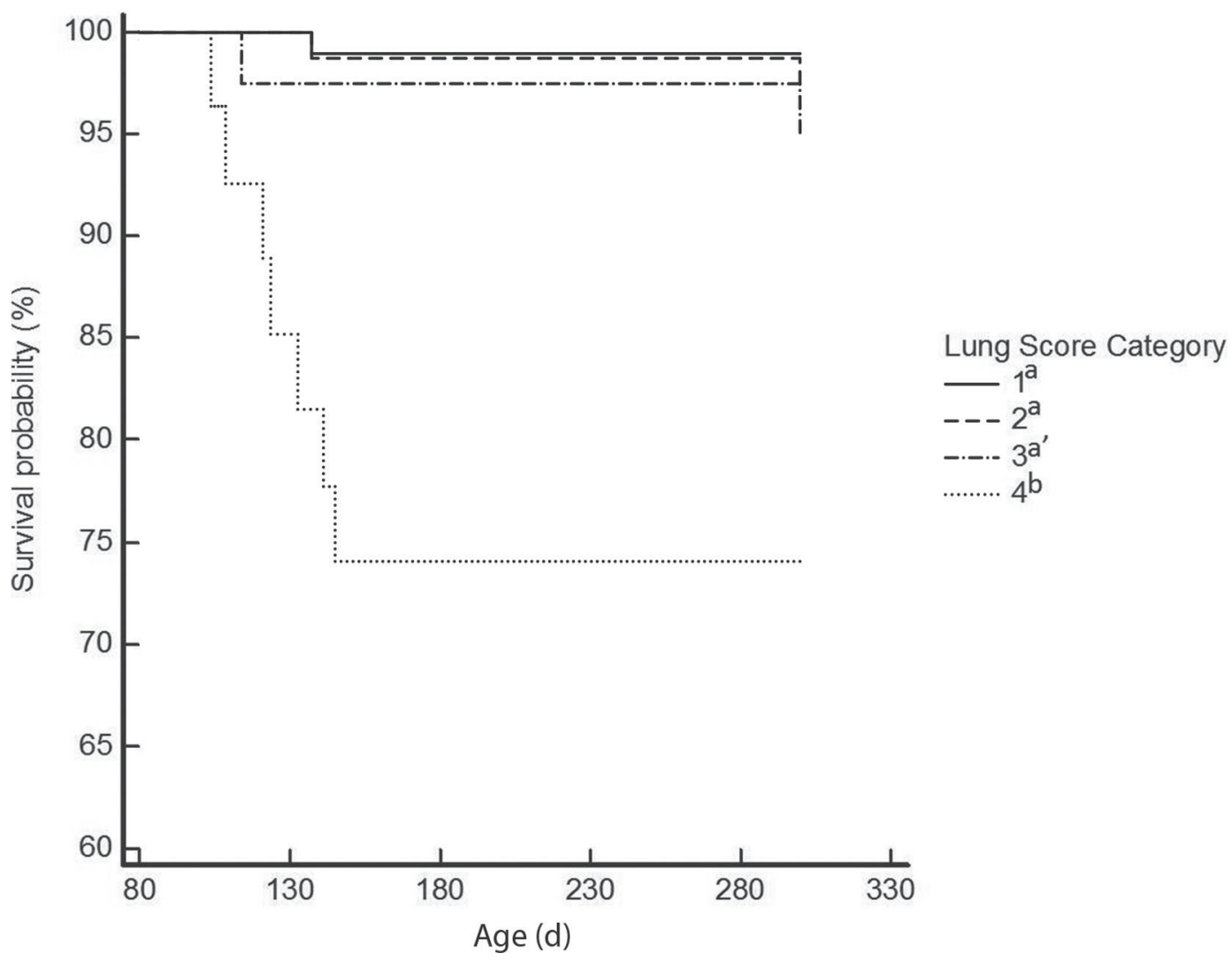

Figure 2. Kaplan-Meier product limit estimates of survival in dairy heifers from 80 to $330 \mathrm{~d}$ of age depending on their ultrasonographic lung consolidation score postweaning. Scores: $1=$ no abnormalities; $2=$ multiple comet tails on the pleural surface or B-lines (coalescence of multiple comet tails without significant lung consolidation); $3=$ one or more location of lung consolidation $>1 \mathrm{~cm}$ but $<6 \mathrm{~cm} ; 4=$ at least one site with $\geq 6 \mathrm{~cm}$ of consolidation, abscessation within the lung parenchyma, or significant pleural effusion $(>1 \mathrm{~cm})$. Different letters $(\mathrm{a}, \mathrm{b})$ indicate a significant difference; the prime symbol (') indicates a tendency comparing score 3 to scores of 1 and 2. 
absence of clinical signs of BRD. This may be helpful to diagnose calves that could benefit from treatment versus those with inactive lung scars that would not have any deleterious effect, and would also help ensure prudent use of antimicrobial drugs.

The present scoring system (1 to 4 scale) was chosen based on an exam that could be performed quickly next to the calf to separate the lesions seen based on presumed severity of findings. The exam is noninvasive, requires 2 min per calf, and can be easily integrated into a physical exam. Many practitioners have incorporated ultrasound into the reproductive services of their practice. The machine used in this study was the same ultrasound and probe that is used routinely for pregnancy diagnosis and fetal sexing.

Other studies have considered the presence of consolidation $\geq 1 \mathrm{~cm}$ in the lung field to be an indicator of BRD (Buczinski et al., 2015). To our knowledge, no consensus exists on the exact ultrasonographic definition of BRD. However, it makes sense that gradation in the scoring system focusing on animals with the most severe lesions should be useful to classify less-productive animals, as previously shown in feedlot animals (Thompson et al., 2006) or veal calves (van der Mei and van den Ingh, 1987). Future studies should look for a more accurate and time-effective scoring system to achieve this goal.

In conclusion, thoracic ultrasound can be easily implemented in a commercial dairy farm setting. When performed at a fixed time, it is helpful to detect calves with a higher probability of experiencing a poor outcome, measured as not entering the first lactation. Thoracic ultrasound provides an objective measurement of lung pathology for BRD, a disease that is subjective to diagnose.

\section{ACKNOWLEDGMENTS}

The authors thank E.I. Medical (Loveland, CO) for their equipment support of this study.

\section{REFERENCES}

Babkine, M., and L. Blond. 2009. Ultrasonography of the bovine respiratory system and its practical application. Vet. Clin. North Am. Food Anim. Pract. 25:633-649.

Bach, A. 2011. Associations between several aspects of heifer development and dairy cow survivability to second lactation. J. Dairy Sci. 94:1052-1057.

Braun, U., N. Pusterla, and M. Fluckiger. 1997. Ultrasonographic findings in cattle with pleuropneumonia. Vet. Rec. 141:12-17.
Buczinski, S., G. Forté, and A. M. Bélanger. 2013. Short communication: Ultrasonographic assessment of the thorax as a fast technique to assess pulmonary lesions in dairy calves with bovine respiratory disease. J. Dairy Sci. 96:4523-4528.

Buczinski, S., T. L. Ollivett, and N. Dendukuri. 2015. Bayesian estimation of the accuracy of the calf respiratory scoring chart and ultrasonography for the diagnosis of bovine respiratory disease in pre-weaned dairy calves. Prev. Vet. Med. 119:227-231.

Flöck, M. 2004. Diagnostic ultrasonography in cattle with thoracic disease. Vet. J. 167:272-280.

Garcia-Peniche, T. B., B. G. Cassell, R. E. Pearson, and I. Misztal 2005. Comparisons of Holsteins with Brown Swiss and Jersey cows on the same farm for age at first calving and first calving interval. J. Dairy Sci. 88:790-796.

Lichtenstein, D., I. Goldstein, E. Mourgeon, P. Cluzel, P. Grenier, and J. J. Rouby. 2004. Comparative diagnostic performances of auscultation, chest radiography, and lung ultrasonography in acute respiratory distress syndrome. Anesthesiology 100:9-15.

McGuirk, S. M. 2008. Disease management of dairy calves and heifers. Vet. Clin. North Am. Food Anim. Pract. 24:139-153.

Ollivett, T., J. Hewson, R. Schubotz, and J. Caswell. 2013. Ultrasonographic progression of lung consolidation after experimental infection with Mannheimia haemolytica in Holstein calves. J. Vet. Intern. Med. 27:673.

Ollivett, T. L., A. J. Burton, R. C. Bicalho, and D. V. Nydam. 2011. Use of rapid thoracic ultrasonography for detection of subclinical and clinical pneumonia in dairy calves. Page 148 in Proc. Am. Assoc. Bovine Pract. Am. Assoc. Bovine Pract., Auburn, AL.

Panciera, R. J., and A. W. Confer. 2010. Pathogenesis and pathology of bovine pneumonia. Vet. Clin. North Am. Food Anim. Pract. $26: 191-214$.

Rabeling, B., J. Rehage, D. Dopfer, and H. Scholz. 1998. Ultrasonographic findings in calves with respiratory disease. Vet. Rec 143:468-471.

Sivula, N., T. Ames, W. Marsh, and R. Werdin. 1996. Descriptive epidemiology of morbidity and mortality in Minnesota dairy heifer calves. Prev. Vet. Med. 27:155-171.

Stanton, A. L., D. F. Kelton, S. J. LeBlanc, J. Wormuth, and K. E. Leslie. 2012. The effect of respiratory disease and a preventative antibiotic treatment on growth, survival, age at first calving, and milk production of dairy heifers. J. Dairy Sci. 95:4950-4960.

Theurer, M. E., B. J. White, R. L. Larson, K. K. Holstein, and D. E. Amrine. 2014. Relationship between rectal temperature at first treatment for bovine respiratory disease complex in feedlot calves and the probability of not finishing the production cycle. J. Am. Vet. Med. Assoc. 245:1279-1285.

Thompson, P. N., A. Stone, and W. A. Schultheiss. 2006. Use of treatment records and lung lesion scoring to estimate the effect of respiratory disease on growth during early and late finishing periods in South African feedlot cattle. J. Anim. Sci. 84:488-498.

USDA-APHIS. 2011. Dairy Heifer Raiser 2011: An overview of operations that specialize in raising dairy heifers. USDA Animal and Plant Health Inspection Service (APHIS), Fort Collins, CO.

van der Mei, J., and T. S. van den Ingh. 1987. Lung and pleural lesions of veal calves at slaughter and their relationship with carcass weight. Vet. Q. 9:203-207.

Van Donkersgoed, J., C. S. Ribble, L. G. Boyer, and H. G. Townsend. 1993. Epidemiological study of enzootic pneumonia in dairy calves in Saskatchewan. Can. J. Vet. Res. 57:247-254.

Wittum, T. E., N. E. Woollen, L. J. Perino, and E. T. Littledike. 1996. Relationships among treatment for respiratory tract disease, pulmonary lesions evident at slaughter, and rate of weight gain in feedlot cattle. J. Am. Vet. Med. Assoc. 209:814-818. 